\title{
Thermo-mechanical and tribological properties of SU-8/h-BN composite with SN150/perfluoropolyether filler
}

\author{
Anand Singh RATHAUR ${ }^{1}$, Jitendra K. KATIYAR ${ }^{2, *}$, Vinay Kumar PATEL ${ }^{1}$ \\ ${ }^{1}$ Department of Mechanical Engineering, Govind Ballabh Pant Institute of Engineering and Technology Ghurdauri, Pauri Garhwal, \\ Uttarakhand 246194, India \\ ${ }^{2}$ Department of Mechanical Engineering, SRM Institute of Science and Technology, Kattankulathur Campus, Chennai, Tamil Nadu \\ 603203, India
}

Received: 05 June 2018 / Revised: 19 September 2018 / Accepted: 15 November 2018

(C) The author(s) 2019.

\begin{abstract}
In this study, SU-8 and its composites are fabricated by blending $10 \mathrm{wt} \%$ hexagonal boron nitride (h-BN) fillers with/without lubricants, such as $10 \mathrm{wt} . \%$ base oil (SN150) and $20 \mathrm{wt} . \%$ perfluoropolyether (PFPE). The thickness of SU-8 and its composites coating is fabricated in the range 100-105 $\mu \mathrm{m}$. Further, SU-8 and its composites are characterized by a 3D optical profilometer, atomic force microscopy, scanning electron microscopy, a thermal gravimetric analyzer, a goniometer, a hardness tester, and an optical microscope. Under a tribology test performed at different normal loads of 2,4 , and $6 \mathrm{~N}$ and at a constant sliding speed of $0.28 \mathrm{~m} / \mathrm{s}$, the reduction in the initial and steady-state coefficient of friction is obtained to be $\sim 0.08$ and $\sim 0.098$, respectively, in comparison to SU-8 $(\sim 0.42$ and $\sim 0.75)$, and the wear resistance is enhanced by more than 103 times that of pure SU-8. Moreover, the thermal stability is improved by $\sim 80-120^{\circ} \mathrm{C}$, and the hardness and elastic modulus by $\sim 3$ and $\sim 2$ times that of pure SU-8, respectively. The SU-8 composite reinforced with $10 \mathrm{wt} . \% \mathrm{~h}-\mathrm{BN}$ and $20 \mathrm{wt} . \%$ PFPE demonstrated the best thermo-mechanical and tribological properties with a nano-textured surface of high hydrophobicity.
\end{abstract}

Keywords: SU-8; boron nitride; perfluoropolyether (PFPE); base oil (SN 150); in-situ lubrication

\section{Introduction}

Surface treatments and coatings have provided tremendous achievements in micro-electro-mechanical system (MEMS) industries from metallurgy to processing [1]. Composite deposits can be coated on steel, light metals, ceramics, plastics, and other materials to enhance the surface properties, such as mechanical, tribological, corrosive, and thermal [2]. For all polymeric materials, SU-8 is a promising material for next generation $3 \mathrm{D}$ fabrication. It can also be used as a coating material in different applications, such as bearing steel [3]. Currently, silicon wafers are used for the fabrication of MEMS devices; however, they have several disadvantages, such as a low thermal stability, difficulty in 3D fabrication, and non-biocompatibility. These disadvantages can be diminished by introducing a polymer, such as SU-8. SU-8 is an epoxy-based negative photo resist that is ultraviolet (UV) curable and can mitigate the disadvantages of a silicon wafer; however, it has poor mechanical and tribological properties [4, 5]. If the mechanical and tribological properties are improved, SU-8 could be used to effectively create small 3D devices. Therefore, researchers have tried to improve these properties by adding solid fillers, liquid fillers, or a solid with liquid fillers. These solid fillers could be a silicon powder, silicon carbide, graphite, graphene, single and multiwall carbon nanotubes, etc., and the liquid fillers could be perfluoropolyether (PFPE), multiply alkylated cyclopentane

* Corresponding author: Jitendra Kumar KATIYAR, E-mail: jitendrakumar.v@ktr.srmuniv.ac.in 
(MAC), SN 150 base oil, mineral oils, etc.

For blending solid fillers in SU-8, Jiguet et al. [6-8] first mixed different weight percentages of silica nano particles. They performed friction and wear tests using a polyoxymethylene (POM) ball and carbon steel ball with a $6 \mathrm{~mm}$ diameter as the interface on the sliding friction apparatus. They observed that wear was reduced by a heat treatment process and an optimal concentration of ( $2.5 \mathrm{wt}$.\%) silica nano particles. They also reported that the coefficient of friction was inversely proportional to the elastic property of SU-8, and the nanoparticles lowered the internal stress of the surface and reduced the coefficient of thermal expansion. Further, Saravanan et al. [9-11] mixed solid fillers, such as graphite, silicon dioxide, and single wall carbon nanotubes in SU-8. They performed a tribology test using a ball-on-disk setup with a $4 \mathrm{~mm}$ diameter silicon nitride ball as the interface. Minimal reduction in friction was observed. Therefore, they further added liquid fillers, such as PFPE, MAC, and SN 150, with solid fillers and found that the coefficient of friction was reduced by approximately 5-6 times, and the wear life was increased by more than $10^{4}$ times. They also performed a nano-indentation test to measure the elastic modulus and hardness and found a marginal increase in the mechanical properties. To continue this study, Katiyar et al. [12-14] mixed carbon fillers, talc, graphite, and a combination of talc and graphite, in different weight percentages in SU-8 and fabricated SU-8 composites. Friction and wear tests were performed on the fabricated composites with a ball-on-disk setup using a silicon nitride ball with a $4 \mathrm{~mm}$ diameter, and the coefficient of friction was $\sim 4-5$ times lower than that of pure SU-8. The mechanical properties of SU-8/talc (30 wt.\%) were $~ 3-4$ times greater than that of pure SU-8.

From the above methods, the solid fillers alone in SU-8 did not show encouraging results in terms of the tribological properties. Therefore, researchers [10, 11] have proposed to mix liquid lubricant fillers with or without solid fillers in SU-8. Liquid lubricant fillers, such as PFPE, MAC, and SN 150 have been mixed with solid fillers, such as CNT and graphite, in an SU-8 matrix, and a tribology test was performed on a ball-on-disk machine. The wear resistance property and wear life were improved by $\sim 10^{5}$ times. Moreover, the initial and steady-state coefficients of friction were reduced. Further, Batooli et al. [15] added ionic liquid in different weight percentages into SU-8 and fabricated an SU-8 composite. These composites were tested on a surface force apparatus (SFA) using a carbon steel ball with a $6 \mathrm{~mm}$ diameter. The friction and wear were directly proportional to the concentration of ionic liquid. Katiyar et al. [16] added PFPE lubricant fillers in different weight percentages with talc and noticed a significant reduction in the coefficient of friction.

Furthermore, from the literature, a nitride $\left(\mathrm{Cr}_{x} \mathrm{~N}\right)$ surface demonstrated high hardness, enhanced wear resistance, and residual compressive stress [17]. Boronbased compounds, such as boron carbide, boron nitride, and transition metal borides, exhibited numerous attractive properties, such as a high melting point and hardness, good wear and corrosion resistance, excellent electrical conductivity, and resistance to attack by molten metals [18]. Hexagonal boron nitride had a graphite-like structure and was suitable for applications where the interface temperature reached $\sim 400{ }^{\circ} \mathrm{C}$. A majority of the studies evaluated the effect of h-BN in clutches, bearing, etc. Kimura et al. [19] mixed h-BN with a lubricant as an additive and performed sliding experiments. They reported that $\mathrm{h}-\mathrm{BN}$ added to a lubricant marginally increased the coefficient of friction and drastically reduced wear. Furthermore, the effect of h-BN on the tribological properties for a sliding bearing was studied by Pawlak et al. [20, 21]. They observed a low coefficient of friction (0.02-0.03) and concluded that the load and pressure-velocity parameters affected the coefficient of friction. They also reported that h-BN was biocompatible and performed in vitro and in vivo tests. Here, h-BN yielded a lower coefficient of friction than that of phospholipids. Chen et al. [22] reported a reduction in the coefficient of friction and an improvement in the wear resistance property after adding h-BN in a copper matrix, which was used for the sleeves of high-speed sewing machinery.

From the published literature, h-BN also acts as a good solid lubricant and provides in-situ lubrication in various applications. The studies have evaluated tribological issues or mechanical issues; however, the tribological and mechanical properties were not simultaneously investigated. Therefore, the main 
objective of this study was to improve the mechanical and tribological properties. Based on this objective, hexagonal boron nitride (h-BN) with and without lubricant fillers SN 150 and PFPE was blended in an SU-8 matrix. To investigate the composites, a thick coating was fabricated, and mechanical and tribological tests were performed on a micro hardness tester and ball-on-disk setup, respectively. Here, h-BN with PFPE enhanced the mechanical and tribological properties.

\section{Experimental procedure}

\subsection{Materials}

The composite coatings of h-BN and h-BN with liquid fillers were fabricated on a $3 \mathrm{~cm} \times 3 \mathrm{~cm}$ glass substrate. SU-8 (Grade-2025, Microchem Ltd USA) was used as the coating material. h-BN (Sigma Aldrich, India) was used in powder form with a particle size of $\sim 1 \mu \mathrm{m}$ (from supplier data). The SU-8 composites were fabricated in two forms: a solid filler, i.e., h-BN, and a solid filler, i.e., h-BN combined with liquid lubricant fillers (SN 150 and PFPE). These lubricant fillers were intended to fabricate a self-lubricating composite. SN-150 (Indian Oil Cooperation Ltd, India), Grade-I, with a viscosity of $4 \mathrm{cSt}$ and Fomblin-Y (also known as functionalized PFPE) (Sigma Aldrich, India) with a viscosity of $60 \mathrm{cSt}$ were used for this research study.

\subsection{Sample preparation}

A glass substrate of $\sim 3 \mathrm{~cm} \times 3 \mathrm{~cm}$ was cleaned using an ultrasonicator (Citizen Scales, India) with deionized water for $\sim 30 \mathrm{~min}$, followed by drying at room temperature $\left(25^{\circ} \mathrm{C}\right)$. The cleaned glass substrate surface was cured by an oxygen plasma cleaner, PDC 32G (Harrick plasma, NY, USA) for $\sim 20 \mathrm{~min}$. The oxygen plasma treatment removed the foreign contaminants and generated the functional group $-\mathrm{OH}$ on the top surface of the glass.

A microbalance (Citizen Scales, India) was used for preparation of the composite solution. Here, $10 \mathrm{wt} . \%$ h-BN was blended, and liquid fillers (SN150 and PFPE) were added $10 \mathrm{wt} . \%$ and $20 \mathrm{wt} . \%$, respectively, to the SU-8 matrix. The volume of the liquid fillers was the same because the density of SN 150 is half the density of PFPE. The composite solution was initially mixed by a stirring rod, followed by an ultrasonic homogenizer (Sonics VCX 130 manufactured by Sonics \& Materials Inc. Newtown, USA) for 25-30 min. The ultrasonic homogenizer prevented agglomeration of the composite solution.

Finally, the prepared mixture of the composites was coated on the glass substrate by a spin coater (S-2000 series MILLMAN, Pune India). The spin coater created a homogeneous coating over the surface and maintained a coating thickness in the range of $\sim 100$ $105 \mu \mathrm{m}$. The prepared coatings were pre-baked at a temperature of $90{ }^{\circ} \mathrm{C}$ for $5-8 \mathrm{~min}$ followed by heating at $100{ }^{\circ} \mathrm{C}$ for $10 \mathrm{~min}$. The pre-baked samples were exposed to UV rays of a $365 \mathrm{~nm}$ wavelength and $210 \mathrm{~mJ} / \mathrm{cm}^{2}$ power for $80-110 \mathrm{~s}$. The UV exposer generated cross linking between the SU-8 molecules. The UV cured samples were post baked at a temperature of $95^{\circ} \mathrm{C}$ for 5-8 min followed by heating at $120{ }^{\circ} \mathrm{C}$ for $10 \mathrm{~min}$. Finally, the prepared samples were placed in a desiccator to protect the prepared samples from foreign contaminations. A similar procedure and parameters were used for preparing the SU-8 composites. The abbreviations used in this study are listed in Table 1.

\subsection{Tribology testing}

Tribology tests were performed on the prepared samples at different normal loads $(2 \mathrm{~N}, 4 \mathrm{~N}$, and $6 \mathrm{~N})$ with a constant sliding speed of $0.28 \mathrm{~m} / \mathrm{s}$ at room temperature, $25^{\circ} \mathrm{C}$, until coating failure or $4 \times 10^{5}$ cycles. The ball-on-disk setup (Ducom Instruments, India) was used to conduct the tribology test using a silicon nitride ball with a $4 \mathrm{~mm}$ diameter and $5 \mathrm{~nm}$ surface roughness $\left(R_{\mathrm{a}}\right)$ (per the supplier's data) at the interface. The silicon nitride ball was used because of its nonreactive nature with most materials and its high hardness. In each test, a new silicon nitride ball was used. For the measurement of friction and wear, a

Table 1 Abbreviations used for the different compositions.

\begin{tabular}{cc}
\hline Materials & Nomenclatures \\
\hline Hexagonal boron nitride & h-BN \\
Pure SU-8 & SU-8 \\
SU-8/BN (10 wt.\%) & BN \\
SU-8/BN (10 wt.\%)/SN150 (10 wt.\%) & BNSN \\
SU-8/BN (10 wt.\%)/PFPE (20 wt.\%) & BNFY \\
\hline
\end{tabular}


full bridge ring shape load cell was used with a calibration factor of $0.75 \mathrm{~N} / \mathrm{mV}$. The load cell was connected to the stain measurement system (SCAD500, Pyrodynamics Ltd., India), followed by the data acquisition system (DAQ, Technocomm Instruments Pvt. Ltd, India) and lab view software (provided by supplier). The friction data are presented in the form of the initial and steady-state coefficients of friction with the standard error. The initial and steady-state coefficients of friction were obtained from the average value of the first 100 sliding cycles and the average value of the last 5,000 sliding cycles, respectively. The average value of the wear life is also presented in this investigation. The wear life showed that the sample contained a visual wear track or the coating failed owing to a fluctuation of the friction. All tests were repeated at least 3 times for each coating sample, and the average value of the data was reported with the standard errors.

\subsection{Mechanical and surface characterization}

The composites were characterized using a 3D optical profilometer, an atomic force microscope, a scanning electron microscope, a thermal gravimetric analyzer, a goniometer, a hardness tester, and an optical microscope.

\subsection{Wettability test}

Wettability tests were performed on the prepared samples before the tribology test using a goniometer (data physics, model: OCA35, 12 VDC, Germany). A volume of $2 \mu \mathrm{l}$ of deionized water was used to measure the contact angle. All tests were performed at room temperature. The tests were repeated six times for each sample, and the average value of the contact angles with the standard error is presented in this study.

\subsection{Thermogravimetric analysis}

The thermal stability of the composite materials was analyzed using a simultaneous thermal analyzer (STA) 8000TGA (PerkinElmer, USA) and is presented by the weight loss with respect to time. The temperature range for the test was maintained between 30 to $550{ }^{\circ} \mathrm{C}$, and the test was performed in the presence of a nitrogen gas environment. The heating rate for the test varied at $10{ }^{\circ} \mathrm{C} \cdot \mathrm{min}^{-1}$, and the cooling rate for test varied at $30{ }^{\circ} \mathrm{C} \cdot \mathrm{min}^{-1}$.

\subsection{Micro-hardness tester}

For measurement of the mechanical properties (elastic modulus and hardness), a micro hardness tester (CSM, Switzerland) was used. Micro indentation was performed using a Vickers indenter with a $50 \mathrm{mN}$ applied load. The indentation depth was in the range of 1-2 $\mu \mathrm{m}$, which was small compared to the coating thickness. For each sample, 4-5 indentations were performed, and the average value of the hardness and elastic modulus with the standard errors are presented.

\subsection{D optical profilometer}

The Contour GTK (Bruker, USA) 3D optical profilometer was used to measure the surface roughness before the tribology test, and after the test, the wear depth and wear volume were observed. Furthermore, the specific wear rate in $\mathrm{mm}^{3} /(\mathrm{N} \cdot \mathrm{m})$ for each coating was measured using the given equation [22].

$$
W_{\mathrm{sp}}=\frac{V}{L D}
$$

Here, $V$ is the wear volume $\left(\mathrm{mm}^{3}\right) ; L$ is the normal load $(\mathrm{N})$, and $D$ is the total sliding distance $(\mathrm{m})$.

\subsection{Optical/scanning electron microscopy}

Finally, the surface morphology of the composite coatings after the tribology test, and the cross-sections of the coatings were observed using a scanning electron microscope (SEM, $\sum$ IGMA, Zeiss, USA). Before obtaining the image from FE-SEM, a titanium coating with a thickness of $\sim 12 \mathrm{~nm}$ at $10 \mathrm{~mA}$ for $75 \mathrm{~s}$ was applied on each sample using a plasma sputter coater (SC7620-CF, Quorum Laughton Lewes). The surface morphology of the composite coatings before the tribology test was also observed using an atomic force microscope, XE7 integrated atomic force microscope (AFM) (Park system, South Korea). The test was performed in a non-contact mode using a silicon cantilever. After the tribology test, the ball interface surface was also evaluated using an optical microscope, Eclipse E100 (Nikon Instruments Inc., USA). 


\section{Results and discussion}

\subsection{Surface characterization}

The surface of the composite material was characterized using a 3D optical profilometer and goniometer. The following results were obtained, as discussed in the following sections.

\subsection{Coating surface}

The coating surface was characterized at a micro- and nano-scale using an optical microscope and atomic force microscopy, respectively. Figures 1(a)-1(d) shows the optical microscopic images of SU-8 and its composite surface. From Fig. 1, more liquid droplets were present in the BNFY composite coating at the same volume than that of BNSN, demonstrating changes in the surface after the addition of fillers. The sizes of these liquid droplets were in the range of $50 \mu \mathrm{m}$ to $1 \mathrm{~nm}$.

The coating surfaces were also observed using AFM, as shown in Figs. 2(a)-2(d). From Fig. 2, the pure SU-8 coating contained a flat surface; however,
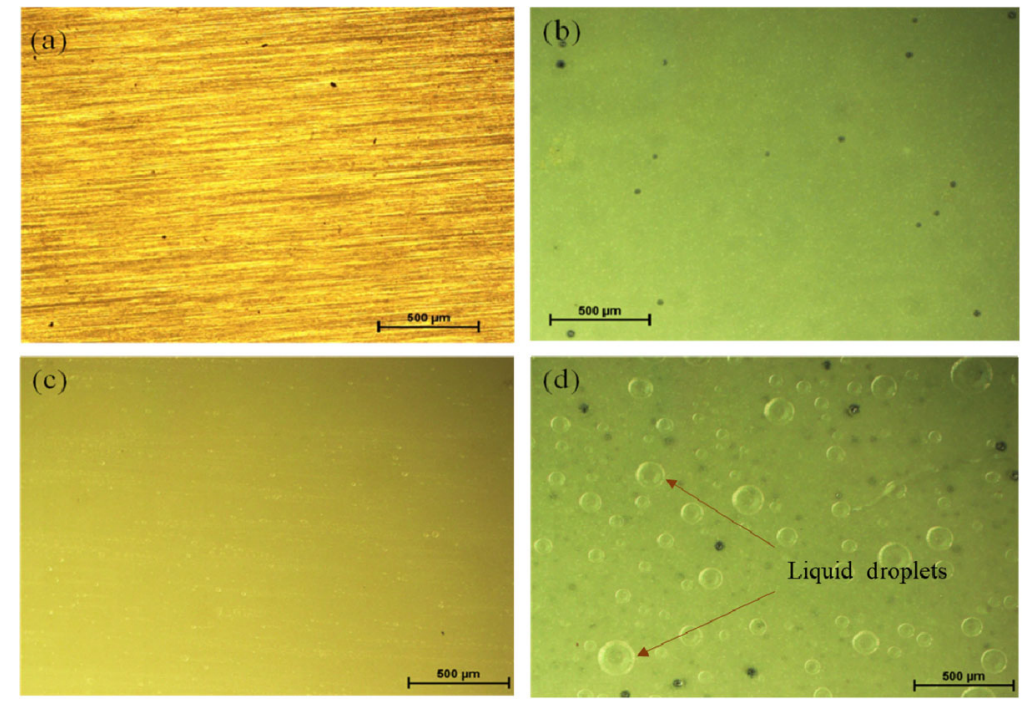

Fig. 1 Optical microscope images of the composite surface: (a) SU-8, (b) BN, (c) BNSN, and (d) BNFY.
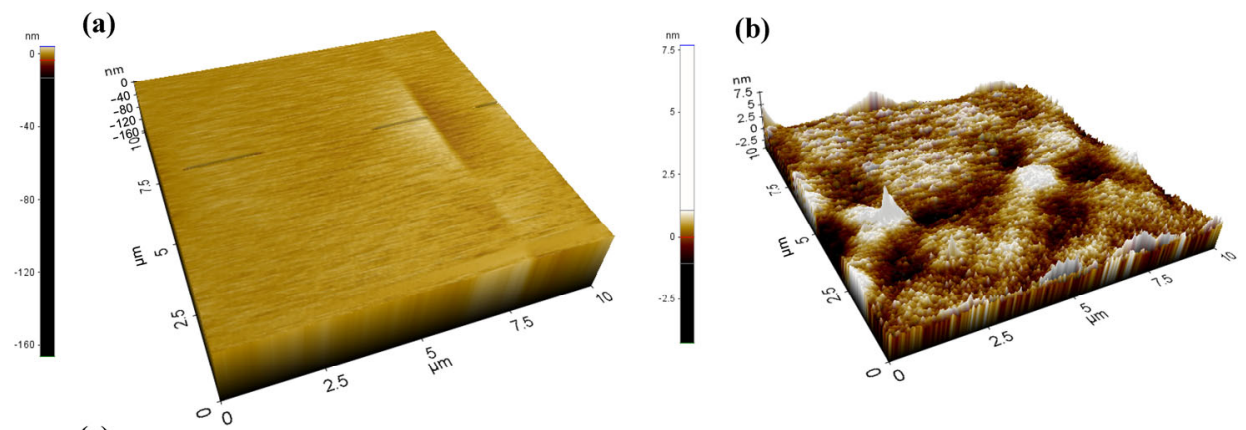

(c)
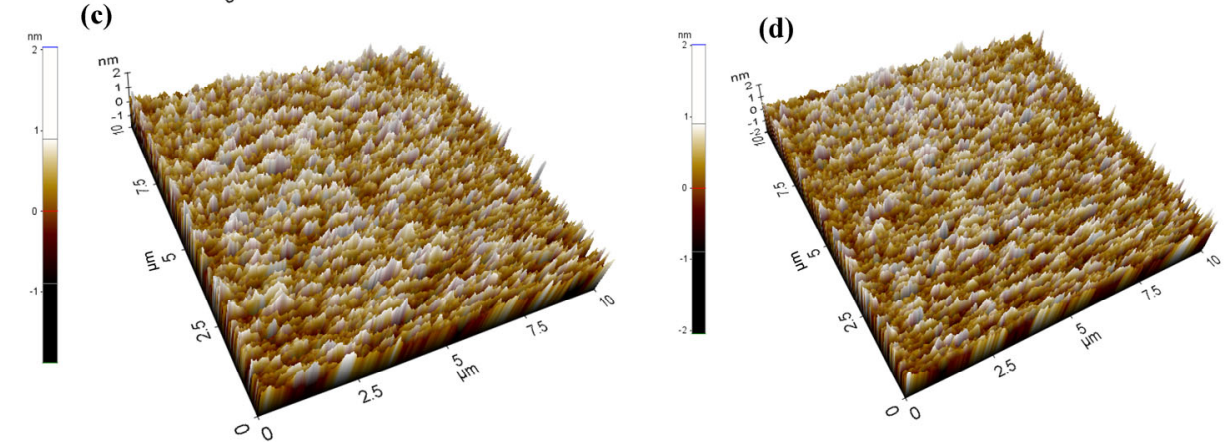

Fig. 2 Atomic force microscope images of the composite surface: (a) SU-8, (b) BN, (c) BNSN, and (d) BNFY. 
after adding the fillers, nano-textures were observed. The surface roughness of the composite coatings was increased. However, after adding the liquid filler (SN 150 or PFPE) in BN, more homogeneous nano-textures were observed over the surface. These homogeneous nano-textures could entrap liquid droplets, which could reduce the coefficient of friction and change the surface from hydrophilic to hydrophobic.

To measure the thickness of the coating and the distribution of the liquid droplets throughout the thickness, scanning electron microscopy was used. The images are shown in Figs. 3(a)-3(d). From Fig. 3, the cross-section of the SU-8 coating surface was smoother than that of the BN composite coating. After adding the liquid fillers in the BN composite coating, the cross-section showed a homogeneous dispersion of the liquid droplets; however, for the BNFY composite, there were more liquid droplets, and the size of the liquid droplets was $\sim 50 \mu \mathrm{m}$ to a few $\mathrm{nm}$. From the figure, the thickness of prepared coating was in the range of $\sim 100-105 \mu \mathrm{m}$.

\subsection{Wettability test}

From the wettability test (contact angle is shown in
Figs. 4(a)-4(d)), the composite surface was changed from hydrophilic to hydrophobic. From Fig. 4, the pure SU-8 and BN coatings were hydrophilic. However, after the addition of the liquid fillers (SN 150 and PFPE), the surfaces of the composites BNSN and BNFY were hydrophobic. The hydrophobic nature of BNFY could be because of the greater surface roughness, as shown in Fig. 5. This phenomenon was also suggested in a previous study; however, they considered different oils and surfaces [23].

\subsection{Roughness test}

Different wear modes were found to link the load to the roughness dependence between the bodies and counter body [24]. The data of the surface roughness with the standard errors are shown in Fig. 5. From Fig. 5, the roughness increased drastically after the addition of the fillers (BN or BN + SN 150/PFPE) in pure SU-8; however, a higher roughness was shown with the BNFY composite, i.e., this composite surface demonstrated a hydrophobic nature. The surface roughness of BNSN was also higher than that of the BN composite. The high surface roughness was owing to the nano-texture, as shown in Figs. 2(b)-2(d).
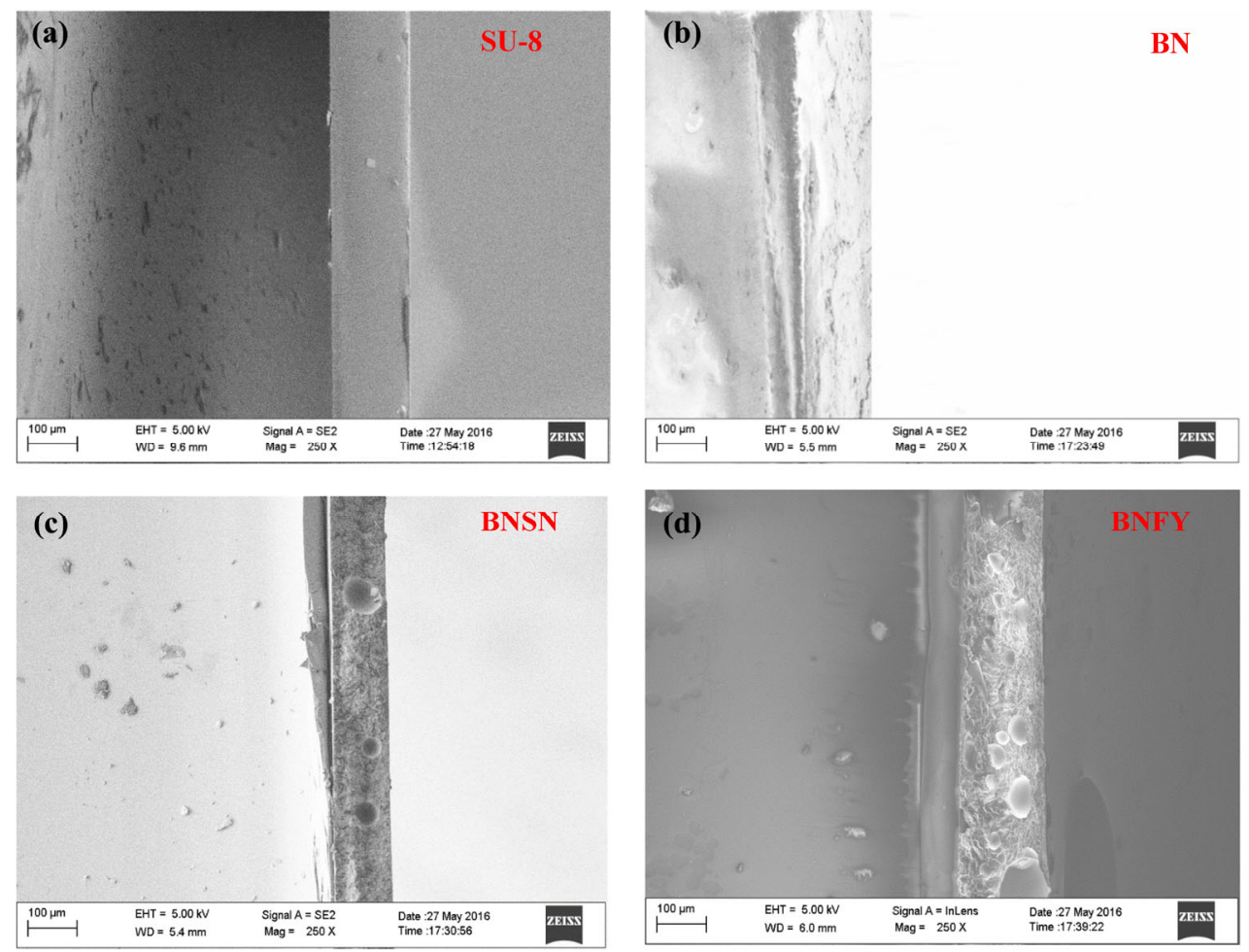

Fig. 3 Cross-sectional images of the composite surface: (a) SU-8, (b) BN, (c) BNSN, and (d) BNFY. 

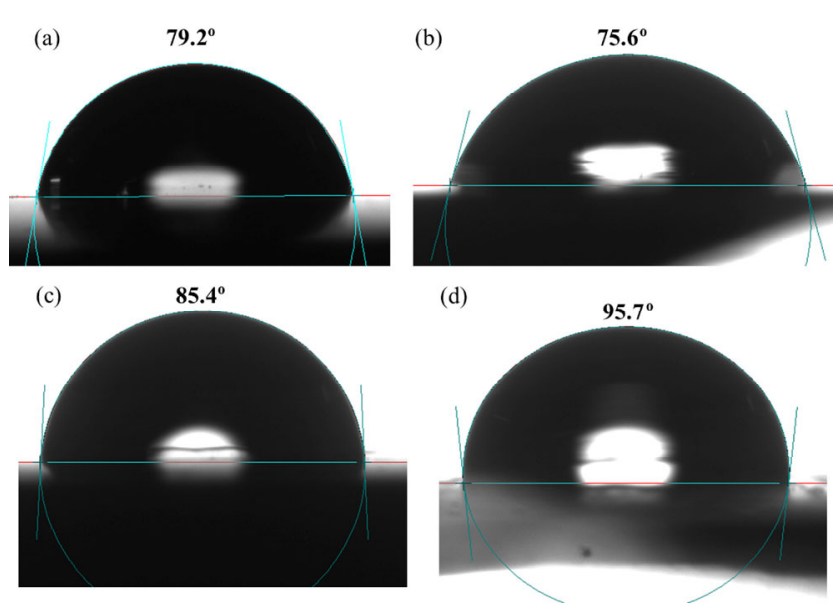

Fig. 4 Contact angle of the composite coating: (a) SU-8, (b) BN, (c) BNSN, and (d) BNFY.

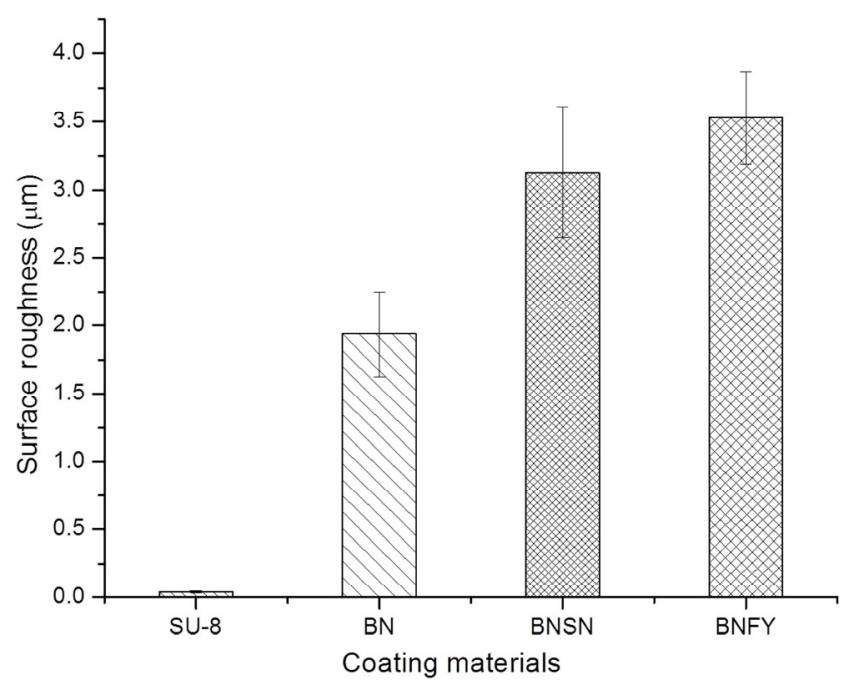

Fig. 5 Surface roughness of the different coating materials.

\subsection{Material characterization}

The material was characterized using a thermogravimetric analyzer and micro harness tester, as stated in the experimental section. A detailed discussion of these characterization methods is given below.

\subsection{Thermogravimetric analysis (TGA)}

The thermal stability of the different composites was measured by a TGA, and the consolidated data of the thermal decomposition are shown in Fig. 6. In all samples, approximately $18 \%$ weight loss was observed at a temperature of $\sim 100{ }^{\circ} \mathrm{C}$. The weight loss at this temperature was owing to the evaporation of the water molecules present in the samples. A significant weight loss in the range of $180-240{ }^{\circ} \mathrm{C}$ was observed.

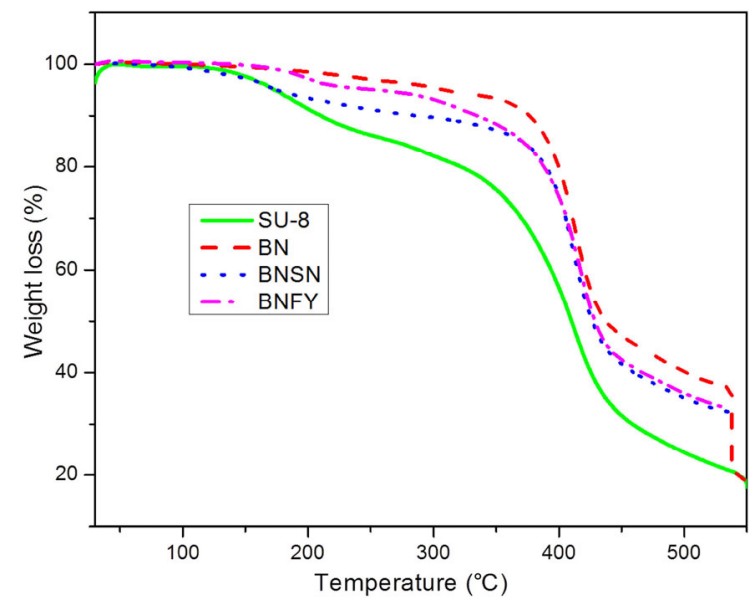

Fig. 6 TGA spectra of SU-8 and its composites.

This weight loss was because of the thermal decomposition of the oxygen carrying functionalities. However, in the liquid carrying samples, the liquid fillers also evaporated at this temperature. Moreover, another major loss began at a temperature of $\sim 280^{\circ} \mathrm{C}$, owing to the decomposition of SU-8 and its composite moieties. The spectra of coating material represented an exothermic weight loss over a wide range of temperatures, $180-500{ }^{\circ} \mathrm{C}$, owing to the slow decomposition. Therefore, the thermal stability of SU-8 was improved by $\sim 80-120{ }^{\circ} \mathrm{C}$ after adding the fillers because of the bonds formed between SU-8 and the fillers.

\subsection{Micro hardness test}

The micro hardness tester was used to measure the elastic modulus and hardness of the prepared coatings. Micro indentation was performed using a Vickers indenter at a $50 \mathrm{mN}$ applied load. The indentation depth was in the range of $\sim 1-2 \mu \mathrm{m}$, which was smaller than the coating thickness. After indentation, the Oliver and Phar method was used to calculate the elastic modulus and hardness of the sample surface, which was incorporated in software supplied by the manufacturer. The consolidated data with the standard error are shown in Figs. 7(a) and 7(b). From Fig. 7(a), the $\mathrm{BN}$ composite contained $\sim 2$ times greater hardness than that of pure SU-8. Similarly, after adding the liquid filler (SN 150), the hardness of the composite was reduced relative to the $\mathrm{BN}$ composite; however, for PFPE, the hardness was marginally increased relative to the $\mathrm{BN}$ composite. Therefore, after adding 

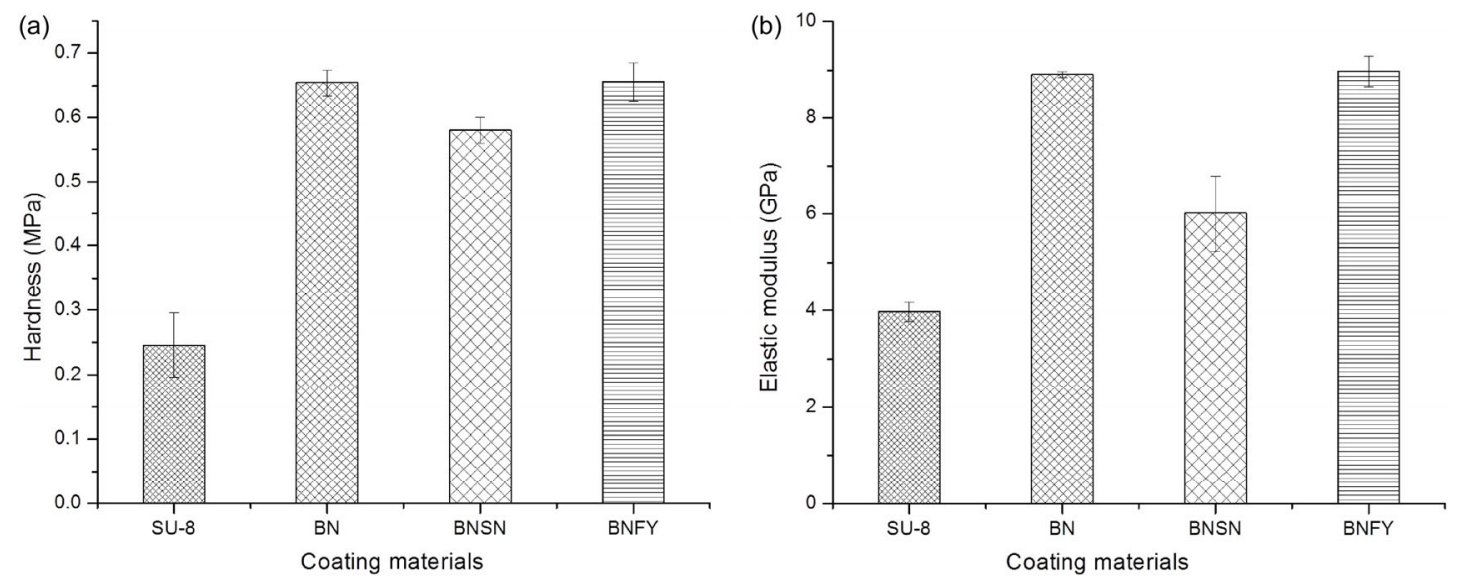

Fig. 7 Mechanical properties of SU-8 and its composite materials: (a) hardness of the coating materials and (b) elastic modulus of the coating materials.

the fillers, the hardness was increased $\sim 3$ times. From Fig. 7(b), the BN and BNFY composites contained a higher elastic modulus ( 2.5 times) than that of pure SU-8. The elastic modulus of the composite affects the Hertzian contact pressure, which is responsible for the friction and wear of the coating. This phenomenon was also discussed by Jiguet et al. [8] with a different material (SU-8/silicon nano-powder composite).

The Hertzian static contact stress is calculated using the following equation [25]

$$
p_{m}=\frac{L}{A}=\frac{L}{\pi\left(\frac{3 R L}{4 E^{*}}\right)^{2 / 3}}
$$

where $L$ is the normal load; $A$ is the Hertzian contact area; $R$ is the radius of the spherical ball, and $E^{*}$ is given as Eq. (3).

$$
E^{*}=\left(\frac{1-v_{1}^{2}}{E_{1}}\right)+\left(\frac{1-v_{2}^{2}}{E_{2}}\right)
$$

Here, $E_{1}$ and $E_{2}$ are the elastic modulus of the two interacting materials, and $v_{1}$ and $v_{2}$ are their Poisson's ratios, respectively. The values of $E$ and the Poisson's ratio $(v)$ for silicon nitride were taken as $310 \mathrm{GPa}$ and 0.27 , respectively, from the supplier data. The Poisson's ratio for SU-8 and its composites was 0.22 [26]. The Hertzian contact stress is listed in Table 2.

\subsection{Friction and wear test}

The friction and wear tests were performed on a
Table 2 Hertzian contact stress (MPa) for the static contact between the $4 \mathrm{~mm}$ diameter silicon nitride ball and coated materials.

\begin{tabular}{cccc}
\hline \multirow{2}{*}{ Materials } & \multicolumn{3}{c}{ Hertzian contact stress (MPa) } \\
\cline { 2 - 4 } & $2 \mathrm{~N}$ & $4 \mathrm{~N}$ & $6 \mathrm{~N}$ \\
\hline SU-8 & 49.57 & - & - \\
BN & 84.13 & - & - \\
BNSN & 65.09 & - & - \\
BNFY & 84.55 & 106.54 & 121.96 \\
\hline
\end{tabular}

ball-on-disk tribometer at a constant sliding speed of $0.28 \mathrm{~m} / \mathrm{s}$ and different loading conditions $(2,4$, and $6 \mathrm{~N}$ ). The raw data of friction for the BNFY composite coating conducted under a $4 \mathrm{~N}$ normal load and $0.28 \mathrm{~m} / \mathrm{s}$ sliding speed at room temperature are shown in Fig. 8. The initial and steady-state coefficients of friction were obtained from the average value of the first 100 sliding cycles and the average value of the last 5,000 sliding cycles, respectively, as shown in Fig. 8. Each tribology test was conducted until failure of the coating or a maximum of $4 \times 10^{5}$ cycles. The average data of the initial and steady-state coefficients of friction are shown in Figs. 9(a) and 9(b) with the standard errors (SE) as error bars based on at least three repetitions for each measurement.

First, the tribology test was conducted at a $2 \mathrm{~N}$ load and $0.28 \mathrm{~m} / \mathrm{s}$ sliding speed for all fabricated composite coatings. At this load, greater initial and steady-state coefficients of friction were observed for pure SU-8. However, after adding the fillers into the SU-8 matrix, the initial coefficients of friction were drastically reduced because the surface of composite became textured, as shown in Fig. 2. These textures 


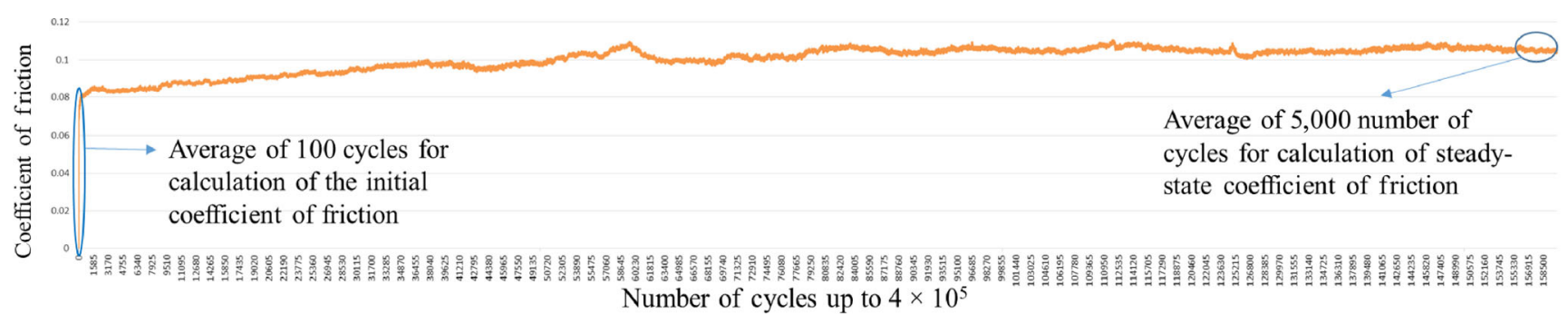

Fig. 8 Raw friction data of the BNFY composite coating at a $4 \mathrm{~N}$ normal load and $0.28 \mathrm{~m} / \mathrm{s}$ sliding speed
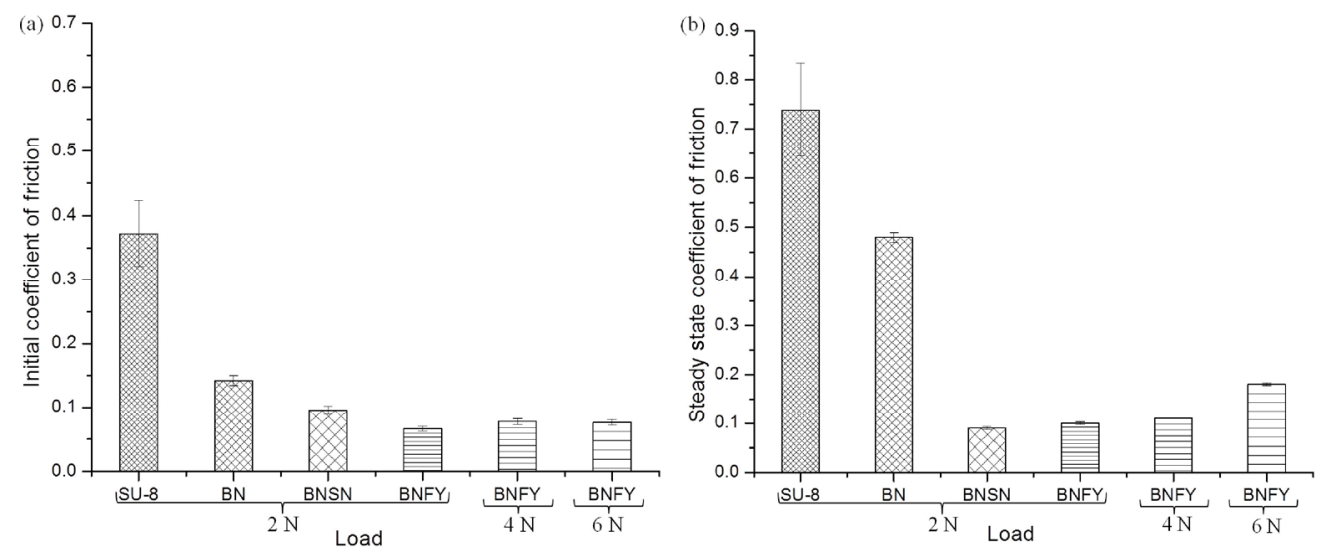

Fig. 9 (a) Effect of the load on the initial coefficient of friction and (b) the effect of the load on the steady-state coefficient of friction for SU-8 and its composites. The error bars in (a) and (b) show the standard errors of three repetitions for each data point.

reduced the contact area. For the $\mathrm{BN}$ composite coating, the initial coefficient of friction was reduced by $\sim 3$ times that of pure SU-8, while the steady-state coefficient of friction was reduced by $\sim 1.5$ times. Moreover, the increase of the steady-state coefficient of friction in the $\mathrm{BN}$ composite was owing to the increase in abrasive wear after $\sim 20,000$ cycles. The abrasive debris were observed at the ball-surface interface after the tribology test, as shown in Fig. 11. After adding the liquid fillers in the BN composite, the BNSN composite contained a low coefficient of friction; however, the coating was not sustained at a $2 \mathrm{~N}$ load. This could be because of the lower hardness and elastic modulus than those of the other composite coatings. Furthermore, the BNFY composite coating showed $\sim 9$ times lower the initial and steady-state coefficients of friction than those of pure SU-8 sample coating, which sustained up to $4 \times 10^{5}$ sliding cycles. Therefore, for further load variation, only the BNFY composite coating was tested because of its excellent properties in terms of the coefficient of friction and wear life. From Fig. 9, the BNFY composite provided lower initial and steady-state coefficients of friction even though the normal load increased from 2 to 4 and $6 \mathrm{~N}$. The low coefficient of friction for the BNFY composite coating could be because of its hydrophobic nature, which reduced adhesion on the surface, and the texture on the surface, which reduced the contact area.

After the tribology test, the surfaces of SU-8 and its composite coatings were characterized by a scanning electron microscope. The images are shown in Figs. 10(a)-10(f). From Fig. 10(a), at $2 \mathrm{~N}$ load, the pure SU-8 sample coating was delaminated and contained a zig-zag shape. This could be because of high contact stress, which caused brittle fracture. This brittlefracture phenomenon was also proposed in a previous investigation using different materials (PTFE/epoxy composite) [27]. After adding the fillers, the delamination of the coating was reduced, and a smooth wear track formed because of the self-lubrication properties of h-BN. Moreover, a homogeneous wear profile was developed, and wear debris were generated after a few cycles. These debris particles rubbed against each other and generated a higher friction than that of the other composites. The BN composite coating showed a three-body wear phenomenon. Therefore, adhesive and abrasive wear occurred in the $\mathrm{BN}$ composite coating. 

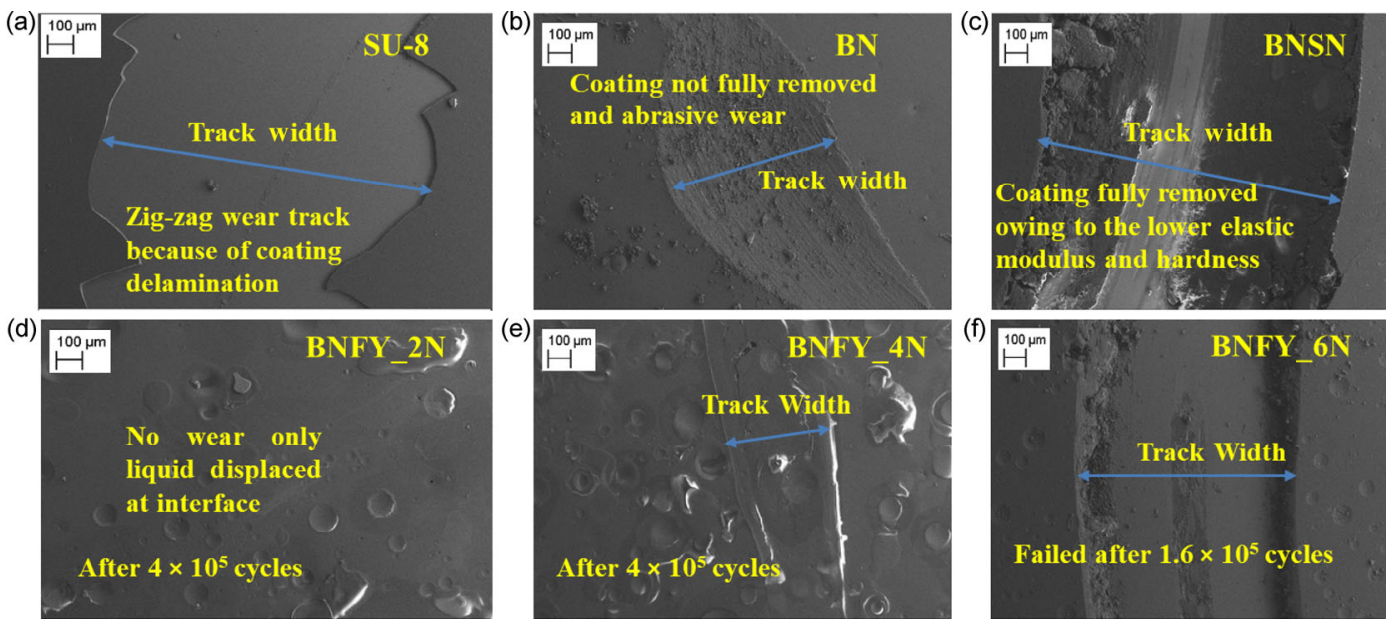

Fig. 10 Optical microscope images of the samples after the tribology test: (a) SU-8 at 2 N, (b) BN at 2 N, (c) BNSN at 2 N, (d) BNFY at $2 \mathrm{~N},(\mathrm{e}) \mathrm{BNFY}$ at $4 \mathrm{~N}$, and (f) BNFY at $6 \mathrm{~N}$. All tests were carried out at a constant sliding speed $(0.28 \mathrm{~m} / \mathrm{s})$.

At a lower load $(2 \mathrm{~N})$, the BNSN composite coating contained lower initial and steady-state coefficients of friction; however, complete removal of the coating occurred. The lower coefficient of friction occurred because of the formation of a lubrication film at the interface. Furthermore, at the lower load, the BNFY composite coating showed negligible wear marks on the surface (Fig. 10(d)) after $4 \times 10^{5}$ cycles. This could be because of the high elastic modulus and in-situ lubrication from the PFPE lubricant inside the matrix. With a further increase in the load from 2 to $4 \mathrm{~N}$, small wear marks were shown in the BNFY composite coating; however, the coating was sustained up to $4 \times$ $10^{5}$ cycles. The wear marks were less because of the lubrication film and texture formation on the coating surface, which reduced the contact area. The surface of BNFY was also sustained at a higher load because this composite coating showed a higher elastic modulus and lower coefficient of friction. This phenomenon was also suggested in a previous study [8] using a different material, such as SU-8 with graphite and a PFPE lubricant. When the load was increased from 4 to $6 \mathrm{~N}$, wear on the coating was present because of the high contact stress ( 1.5 times greater) at the interface; however, the wear tack was smooth owing to the in-situ lubrication at the interface.

For confirmation of the wear phenomenon, ball interface images were obtained using a microscope, as shown in Figs. 11 (a)-1(f). Figure 11(a) shows that the coating was delaminated because of brittle fracture, and the delaminated coating parts are represented by an arrow. Furthermore, the BN composite coating showed initial adhesive wear followed by abrasive wear (Fig. 11(b)). However, the BNSN composite demonstrated ploughing of the coating and caused ploughing wear (shown in Fig. 11(c)). Moreover, from Fig. 11(d), a large number of liquid molecules of PFPE in the form of droplets were present at the interface, providing in-situ lubrication at the interface. Therefore, wear of the material did not occur. When the load increased from 2 to $4 \mathrm{~N}$, the quantity of liquid lubricant was reduced because of the high heat generated at the interface by increasing the load. This caused mild wear marks on the surface, as shown in Fig. 10(e).

Moreover, after increasing the load from 4 to $6 \mathrm{~N}$, adhesive and abrasive wear developed owing to the high contact pressure; however, these abrasive particles consisted of PFPE lubricant, which reduced the coefficient of friction at a higher load.

The wear test (also known as a long-term friction test) was performed on SU-8 and its composites until failure of the coating or a maximum $4 \times 10^{5}$ sliding cycles. The wear-rate of the coating was calculated by Eq. (1), explained in experiment section, and the data with the SEs are shown in Fig. 12.

From Fig. 12, at a lower load, the BNFY composite showed lower wear resistance than that of pure SU-8 and other composite coating materials. The lower wear rate of the BNFY composite was owing to the in-situ lubrication film at the interface (Figs. 9 and 10(d)), higher elastic modulus, and hydrophobic nature of the coating surface. After increasing the load at the 
(a)
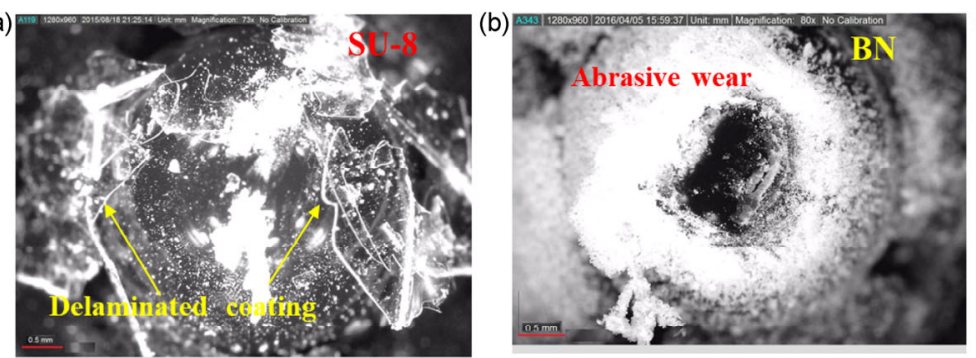

(d)

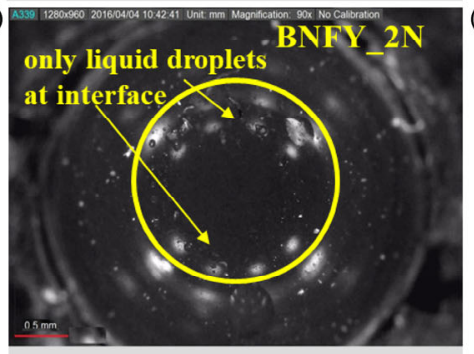

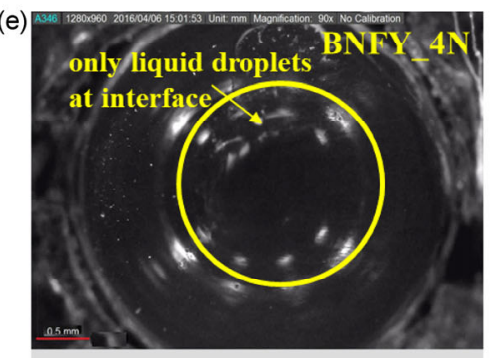
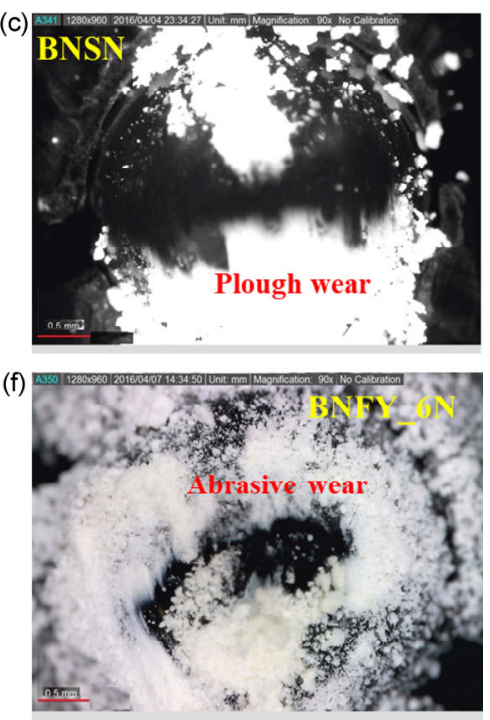

Fig. 11 Portable microscope images of the ball after the tribology test: (a) SU-8 at $2 \mathrm{~N}$, (b) BN at $2 \mathrm{~N}$, (c) BNSN at $2 \mathrm{~N}$, (d) BNFY at $2 \mathrm{~N}$, (e) BNFY at $4 \mathrm{~N}$, and (f) BNFY at $6 \mathrm{~N}$. All tests were carried out at a constant sliding speed $(0.28 \mathrm{~m} / \mathrm{s})$. The scale is $0.5 \mathrm{~mm}$ in each image.

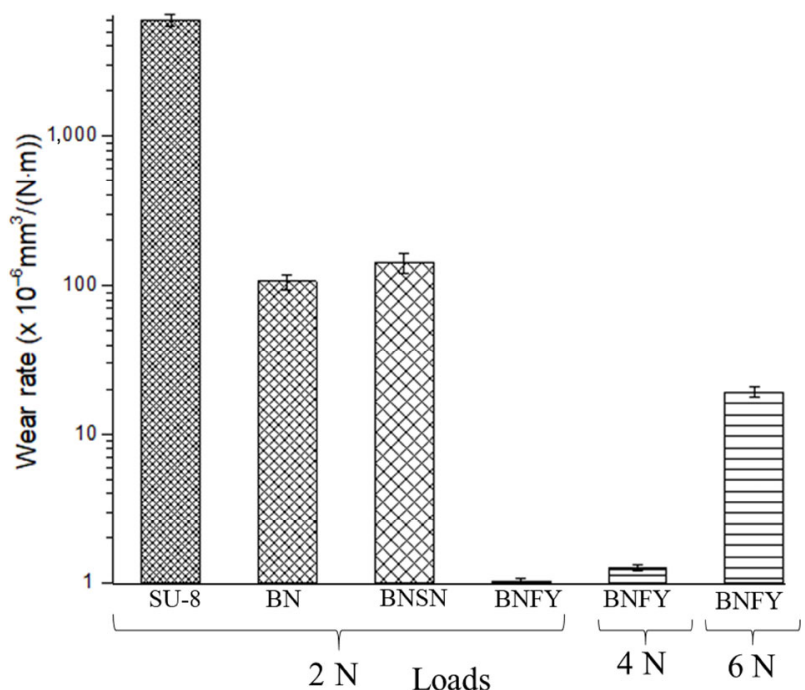

Fig. 12 Wear rate of SU-8 and its composites until failure of the coating at different normal loads and a constant $0.28 \mathrm{~m} / \mathrm{s}$ sliding speed.

interface, the wear rate slightly increased owing to an increase in the contact pressure at the interface, which increased the frictional heating at the interface. Furthermore, with an increase of the load from 4 to $6 \mathrm{~N}$, the formation of the in-situ lubrication film for the BNFY composite at the interface was reduced, which caused abrasive wear and changed the wear phenomenon from two body to three body. This increased the wear rate by $\sim 19$ times that of the same coating materials tested at a $2 \mathrm{~N}$ load. Therefore, at a lower load (2 N), the BNFY composite showed a high wear resistance property, greater than $10^{3}$ times that of pure SU-8.

\section{Conclusions}

From this study, the following conclusions are drawn.

1. After adding the fillers, textures were generated on the surface, which changed the surface from hydrophilic to hydrophobic.

2. The thermal stability was improved by $80-120{ }^{\circ} \mathrm{C}$ owing to the bonds formed between SU-8 and the fillers. Moreover, the hardness was $\sim 3$ times higher and the elastic modulus by $\sim 2.5$ times higher than those of pure SU-8.

3. For the friction test, at a lower load $(2 \mathrm{~N})$, the composite fillers showed a lower coefficient of friction than that of pure SU-8. However, after adding h-BN, the initial and steady-state coefficients of friction were reduced by $\sim 3$ times and $\sim 2$ times, respectively. Moreover, after adding lubricant fillers with h-BN, the initial and steady-state coefficients of friction were reduced by $\sim 8$ times and $\sim 9$ times, respectively.

4. At a higher load, the BNFY composite yielded lower initial and steady-state coefficients of friction than those of pure SU-8.

5. From the wear test, the BNFY composite showed a high wear resistance property, $10^{3}$ times greater than that of pure SU-8. However, after adding the fillers, delamination of the coating was reduced, and a smooth 
wear track was formed. In addition, the BNFY composite showed negligible wear marks on the surface after $4 \times 10^{5}$ cycles at a low load $(2 \mathrm{~N})$.

The SU-8 composite with $10 \mathrm{wt} . \% \mathrm{BN}$ and $20 \mathrm{wt} . \%$ PFPE lubricant provided a low coefficient of friction, high wear resistance property, and high wear life at low loads. Moreover, the composite coating improved the mechanical and surface properties. Therefore, the composite coating could be useful for bearing applications and MEMS fabrication.

Furthermore, this work could be extended to optimize the coating thickness for lower load and higher load applications.

Open Access: This article is licensed under a Creative Commons Attribution 4.0 International License, which permits use, sharing, adaptation, distribution and reproduction in any medium or format, as long as you give appropriate credit to the original author(s) and the source, provide a link to the Creative Commons licence, and indicate if changes were made.

The images or other third party material in this article are included in the article's Creative Commons licence, unless indicated otherwise in a credit line to the material. If material is not included in the article's Creative Commons licence and your intended use is not permitted by statutory regulation or exceeds the permitted use, you will need to obtain permission directly from the copyright holder.

To view a copy of this licence, visit http://creativecommons.org/licenses/by/4.0/.

\section{References}

[1] Bertsch A, Renaud P. Special issue: 15 years of SU8 as MEMS material. Micromachines 6(6): 790-792 (2015)

[2] Steinhäuser S, Wielage B. Composite coatings: Manufacture, properties, and applications. Surf Eng 13(4): 289-294 (1997)

[3] Rathaur A S, Katiyar J K, Patel V K, Bhaumik S, Sharma A K. A comparative study of tribological and mechanical properties of composite polymer coatings on bearing steel. Int J Surf Sci Eng 12(5-6): 379-401 (2018).

[4] Lau K H, Giridhar A, Harikrishnan S, Satyanarayana N, Sinha S K. Releasing high aspect ratio SU-8 microstructures using AZ photoresist as a sacrificial layer on metallized $\mathrm{Si}$ substrates. Microsyst Technol 19(11): 1863-1871 (2013)

[5] Maboudian R, Ashurst W R, Carraro C. Tribological challenges in micromechanical systems. Tribol Lett 12(2): 95-100 (2002)

[6] Jiguet S, Judelewicz M, Mischler S, Hofmann H, Bertsch A, Renaud P. SU-8 nanocomposite coatings with improved tribological performance for MEMS. Surf Coat Technol 201(6): 2289-2295 (2006)

[7] Jiguet S, Judelewicz M, Mischler S, Bertch A, Renaud P. Effect of filler behavior on nanocomposite SU8 photoresist for moving micro-parts. Microelectron Eng 83(4-9): 1273-1276 (2006)

[8] Jiguet S, Bertsch A, Judelewicz M, Hofmann H, Renaud P. SU-8 nanocomposite photoresist with low stress properties for microfabrication applications. Microelectron Eng 83(10): 1966-1970 (2006)

[9] Saravanan P, Satyanarayana N, Sinha S K. Self-lubricating SU-8 nanocomposites for microelectromechanical systems applications. Tribol Lett 49(1): 169-178 (2013)

[10] Saravanan P, Satyanarayana N, Sinha S K. Wear durability study on self-lubricating SU-8 composites with perfluoropolyther, multiply-alkylated cyclopentane and base oil as the fillers. Tribol Int 64: 103-115 (2013)

[11] Saravanan P, Satyanarayana N, Siong P C, Duong H M, Sinha S K. Tribology of self-lubricating SU-8+PFPE composite based Lub-tape. Procedia Eng 68: 497-504 (2013)

[12] Katiyar J K, Sinha S K, Kumar A. Effects of carbon fillers on the tribological and mechanical properties of an epoxy-based polymer (SU-8). Tribol-Mater, Surf Interfaces 10(1): 33-44 (2016)

[13] Katiyar J K, Sinha S K, Kumar A. Effect of graphite concentration on the tribological and mechanical properties of filled SU-8 polymer. Tribol Online 11(2): 152-158 (2016)

[14] Katiyar J K, Sinha S K, Kumar A. Friction and wear durability study of epoxy-based polymer (SU-8) composite coatings with talc and graphite as fillers. Wear 362-363: 199-208 (2016)

[15] Batooli L, Maldonado S G, Judelewicz M, Mischler S. Novel SU-8/ionic liquid composite for tribological coatings and MEMS. Micromachines 6(5): 611-621 (2015)

[16] Katiyar J K, Sinha S K, Kumar A. In situ lubrication of SU-8/talc composite with base oil (SN150) and perfluoropolyether as fillers. Tribol Lett 64(1): 5 (2016)

[17] Basu A, Majumdar J D, Manna I. Structure and properties of $\mathrm{Cr}_{\mathrm{x}} \mathrm{N}$ coating. Surf Eng 28(3): 199-204 (2012)

[18] Dearnley P A, Bell T. Engineering the surface with boron based materials. Surf Eng 1(3): 203-217 (1985)

[19] Kimura Y, Wakabayashi T, Okada K, Wada T, Nishikawa H. Boron nitride as a lubricant additive. Wear 232(2): 199-206 (1999)

[20] Pawlak Z, Kaldonski T, Pai R, Bayraktar E, Oloyede A. A comparative study on the tribological behaviour of hexagonal 
boron nitride (h-BN) as lubricating micro-particles-An additive in porous sliding bearings for a car clutch. Wear 267(5-8): 1198-1202 (2009)

[21] Pawlak Z, Pai R, Bayraktar E, Kaldonski T, Oloyede A. Lamellar lubrication in vivo and vitro: friction testing of hexagonal boron nitride. Biosystems 94(3): 202-208 (2008)

[22] Chen S Y, Bi Y N, Zhang H, Liang J, Wellburn D, Liu C S. Effect of BN fraction on the mechanical and tribological properties of $\mathrm{Cu}$ alloy/BN self-lubricating sleeves. J Compos Mater 49(30): 3715-3725 (2015)

[23] Broje V, Keller A A. Interfacial interactions between hydrocarbon liquids and solid surfaces used in mechanical oil spill recovery. J Colloid Interface Sci 305(2): 286-292 (2007)

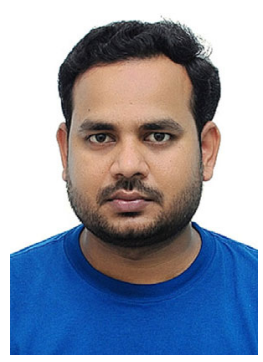

Anand Singh RATHAUR. He received his M.Tech degree from Swami Vivekananda Subharti University, Meerut Uttar Pradesh. Presently, he is doing doctorate from

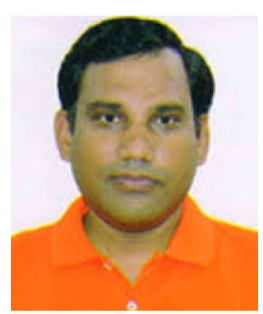

Jitendra Kumar KATIYAR. He is research assistant professor in the Department of Mechanical Engineering, SRM Institute of Science and Technology, Kattankulathur, Tamil Nadu, India. His research interests include tribology of carbon materials, polymer composites, self-lubricating polymers, lubrication tribology and coatings for advanced technologies. He obtained his Ph.D. degree from the Indian Institute of Technology Kanpur in 2017 and master degree

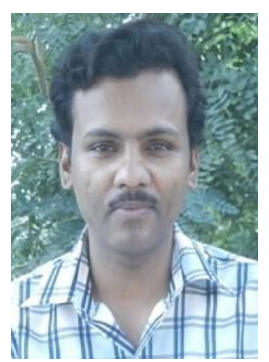

Vinay K. PATEL. He is assistant professor in the Department of Mechanical Engineering, Govind Ballabh Pant Institute of Engineering and Technology, Pauri Garhwal, Uttarakhand, India. He completed his Ph.D. degree from Indian
[24] Celis J P, Stals L, Vancoille E, Mohrbacher H. Wear testing of hard coatings: more than wear rate. Surf Eng 14(3): 205210 (1998)

[25] Johnson K L. Contact Mechanics. Cambridge (UK): Cambridge University Press, 1985

[26] Myint S M, Minn M, Ren Y P, Satyanarayana N, Sinha S K, Bhatia C S. Friction and wear durability studies on the 3D negative fingerprint and honeycomb textured SU-8 surfaces. Tribol Int 60: 187-197 (2013)

[27] McCook N L, Burris D L, Kim N H, Sawyer W G. Cumulative damage modeling of solid lubricant coatings that experience wear and interfacial fatigue. Wear 262(11-12): 1490-1495 (2007)

Govind Ballabh Pant Institute of Engineering and Technology, Pauri, Garhwal, Uttarakhand. His areas of interest are polymer composite, composite coatings and tribology.

from the same institution in 2010. He obtained his bachelor degree from UPTU Lucknow with Honors in 2007. He has several professional memberships such as Tribology Society of India, Malaysian Society of Tribology, and The Indian Society for Technical Education (ISTE). He has published more than two dozen papers in reputed journals and international conferences. He is also a reviewer in many reputed journals such as Tribology International, Friction, Material Research Express, and Inderscience Journals.

Institute of Technology Kanpur in 2015, specializing in nano-energetic materials. His research interests include nano-energetic materials, MEMS, welding and tribology. He has published 25 peer-reviewed journal articles, 7 conference papers, 8 chapters and edited one book on nano-energetic materials. 